\title{
An Update on the 0Z Project
}

\author{
J. G. Cohen*, N. Christlieb ${ }^{*}$, A. McWilliam**, S. Shectman** and I. Thompson** \\ ${ }^{*}$ California Institute of Technology \\ ${ }^{\dagger}$ Uppsala University, Sweden \\ ${ }^{* *}$ Carnegie Observatories of Washington
}

\begin{abstract}
.
We give an update on our OZ Survey to find more extremely metal poor (EMP) stars with $[\mathrm{Fe} / \mathrm{H}]<-3$ dex through mining the database of the Hamburg/ESO Survey. We present the most extreme such stars we have found from $\sim 1550$ moderate resolution follow up spectra. One of these, HE1424-0241, has highly anomalous abundance ratios not seen in any previously known halo giant, with very deficient $\mathrm{Si}$, moderately deficient $\mathrm{Ca}$ and $\mathrm{Ti}$, highly enhanced $\mathrm{Mn}$ and $\mathrm{Co}$, and low $\mathrm{C}$, all with respect to $\mathrm{Fe}$. We suggest a SNII where the nucleosynthetic yield for explosive $\alpha$-burning nuclei was very low compared to that for the hydrostatic $\alpha$-burning element $\mathrm{Mg}$, which is normal in this star relative to Fe. A second, less extreme, outlier star with high $[\mathrm{Sc} / \mathrm{Fe}]$ has also been found

We examine the extremely metal-poor tail of the HES metallicity distribution function (MDF). We suggest on the basis of comparison of our high resolution detailed abundance analyses with $[\mathrm{Fe} / \mathrm{H}](\mathrm{HES})$ for stars in our sample that the MDF inferred from follow up spectra of the HES sample of candidate EMP stars is heavily contaminated for $[\mathrm{Fe} / \mathrm{H}](\mathrm{HES})<-3$ dex; many of the supposed EMP stars below that metallicity are of substantially higher Fe-metallicity, including most of the very C-rich stars, or are spurious objects.
\end{abstract}

Keywords: stellar abundances, Galactic halo, extremely metal poor stars PACS: $97.10 . \mathrm{Tk}$

\section{INTRODUCTION}

Extremely metal poor (EMP) stars provide important clues to the chemical history of our Galaxy, the role and type of early SN, the mode of star formation in the proto-Milky Way, and the formation of the Galactic halo. The sample of known EMP stars is summarized by [1] They compiled a list of the key properties of the 12 stars identified up to that time with $[\mathrm{Fe} / \mathrm{H}] \leq-3.5 \mathrm{dex}, 7$ of which are EMP giants and subgiants within the range of $T_{\text {eff }}$ between 4900 and $5650 \mathrm{~K}$

Our $0 \mathrm{Z}$ project has the goal of increasing the sample of such stars through data mining of the Hamburg/ESO Survey [2]. This is an objective prism survey from which it is possible to efficiently select QSOs [2] as well as a variety of interesting stellar objects, among them EMP stars [3].

Our $0 \mathrm{Z}$ project has been systematically searching the database of the HES for this purpose over the past five years. We have just published [4], and we present at this conference, a sample of new EMP giants with $T_{\text {eff }}<$ $6000 \mathrm{~K}$ and $[\mathrm{Fe} / \mathrm{H}] \leq-3.5$ dex which substantially increases the number of such stars known.

\section{OUTLIERS}

Our $0 \mathrm{Z}$ project has obtained moderate resolution follow up spectra of $\sim 1550$ candidate EMP stars selected from the HES. These follow up spectra were processed through the code described in [5], which is essentially identical to that used by the HK Survey [6] until recently; the latest updates to the algorithm as used by the HK Survey are described in [7]. The algorithm uses the strength of $\mathrm{H} \delta$ and of the $3933 \AA \mathrm{Ca}$ II line to assign a metallicity to each star, which we denote as $[\mathrm{Fe} / \mathrm{H}](\mathrm{HES})$. (The SEGUE survey uses an even more sophisticated algorithm taking advantage of the uniform wide wavelength coverage of the SDSSII spectra and multi-color photometry; a preliminary description is given in [8].) We attempt to observe all stars with $[\mathrm{Fe} / \mathrm{H}](\mathrm{HES})<-2.9 \mathrm{dex}$ as inferred from these moderate resolution follow up spectra at high resolution.

We present the results of detailed abundance analyses based on high resolution and high signal-to-noise spectra of eight extremely metal poor (EMP) stars with $[\mathrm{Fe} / \mathrm{H}]$ $\leq-3.5 \mathrm{dex}$, four of which are new. Only stars with $4900<T_{\text {eff }}<5650 \mathrm{~K}$ are included.

Past work on EMP stars, e.g. [9], [10] and [11], has emphasized the small scatter at a fixed $[\mathrm{Fe} / \mathrm{H}]$ for trends of element abundance ratios $[\mathrm{X} / \mathrm{Fe}]$. The $\sigma$ about the mean at each $[\mathrm{Fe} / \mathrm{H}]$ appears to be consistent with very little intrinsic scatter, just the observational errors contributing. This applies only to $\mathrm{C}$-normal metal-poor stars, and to elements between $\mathrm{Na}$ and the end of the Fe-peak. The light elements Li, Be, C, N, and $\mathrm{O}$ in EMP first ascent RGB stars may be affected by mixing of internally processed material, while EMP AGB stars may have Na 
and $\mathrm{Al}$ affected as well [12]. We established in [13] that the neutron capture elements are decoupled from the $\mathrm{Fe}$ peak elements; the site or conditions of their production, presumably via the $r$ or $s$-process, must be different from that of the Fe-peak elements.

The most interesting thing we have discovered recently is the existence of outliers in these relations among EMP stars. The most sensational case we have found, for which a brief description was given in [14] with a full study in [4], is HE1424-0241. This star is the most metal poor star in our sample, with $[\mathrm{Fe} / \mathrm{H}] \sim-4 \mathrm{dex}$. It has highly anomalous abundance ratios unlike those of any other known EMP giant, with very low $\mathrm{Si}, \mathrm{Ca}$ and Ti relative to $\mathrm{Fe}$, and enhanced $\mathrm{Mn}$ and $\mathrm{Co}$, again relative to Fe. Figs. 1 and 2 illustrate the uniqueness of this star among other halo giants with regard to its $\mathrm{Ca}$ and $\mathrm{Si}$ abundance ratios with respect to Fe. Only (low) upper limits for $\mathrm{C}$ and $\mathrm{N}$ can be derived from the non-detection of the $\mathrm{CH}$ and $\mathrm{NH}$ molecular bands.

$\mathrm{Si}, \mathrm{Ca}$ and $\mathrm{Ti}$ are formed via explosive $\alpha$-burning while $\mathrm{Mg}$, which is normal with respect to $\mathrm{Fe}$ in HE1424-0241, is formed via hydrostatic $\alpha$-burning. A much smaller separation of abundance ratios $(\sim 0.2 \mathrm{dex})$ for elements between these two types of $\alpha$-burning was seen by [15] in the Galactic bulge, but that of HE1424-0241 is much more extreme, and cannot be explained with recent SN nucleosynthesis yields. We suggest a peculiar SNII produced the chemical inventory of this star. HE0132-2429, another sample star, has excesses of $\mathrm{N}$ and $\mathrm{Sc}$ with respect to $\mathrm{Fe}$.

The strong outliers in abundance ratios among the Fe-peak elements in these C-normal stars, not found at somewhat higher metallicities $([\mathrm{Fe} / \mathrm{H}] \sim-3 \mathrm{dex})$, are definitely real. The anomalies discussed here cannot be due to observational or analysis errors. There are two HIRES spectra for each of these two stars. The effects are very large, and are measured relative to the many other EMP stars we have analyzed in a homogeneous manner with identical codes and procedures.

They suggest that at such low metallicities we are beginning to see the anticipated and long sought stochastic effects of individual supernova events contributing to the Fe-peak material within a single star.

Another of our recent results [see 4] comes from a detailed comparison of the analysis procedures adopted by our $0 Z$ project compared to those of the First Stars VLT Large Project. We find a systematic difference for $[\mathrm{Fe} / \mathrm{H}]$ of $\sim 0.3 \mathrm{dex}$, our values always being higher, largely due to differences in adopted reddenings, temperature scales, and Fe solar abundance between the two projects.

\section{THE METALLICITY DISTRIBUTION FUNCTION OF THE HES}

A preliminary metallicity distribution function for the HES was published in [1]. We examine here the likely validity of this result, focusing on the most extreme of the EMP stars. We ignore issues of incompleteness, which are currently being studied by $\mathrm{T}$. Schörck and N. Christlieb, and which while small at $[\mathrm{Fe} / \mathrm{H}]<3 \mathrm{dex}$, become quite large by -2 dex.

Our $0 \mathrm{Z}$ project has obtained moderate resolution follow up spectra of $\sim 1550$ candidate EMP stars selected from the HES. We have attempted to observe all stars with $[\mathrm{Fe} / \mathrm{H}](\mathrm{HES})<-2.9 \mathrm{dex}$ as inferred from these follow up spectra at high resolution. We list below the 12 objects from the $0 Z$ project for which the resulting $[\mathrm{Fe} / \mathrm{H}](\mathrm{HES}$ ) was less than $-3.5 \mathrm{dex}$. (The ID is truncated for stars whose analyses we have not yet published.)

Since these are in principle stars of great interest, the follow up spectra were scrutinized with care. Three of the 12 were eliminated in that way; two are $M$ dwarfs and one is a QSO. Each object that survived (with one exception) has now been observed at high resolution with either HIRES at Keck or MIKE at Magellan.

Of the three objects predicted to have $[\mathrm{Fe} / \mathrm{H}]<$ $-4 \mathrm{dex}$, none have $[\mathrm{Fe} / \mathrm{H}](\mathrm{HIRES})<-2.8 \mathrm{dex}$. This leaves 8 of the 12 objects. Three of these are C-stars, two of them have $[\mathrm{Fe} / \mathrm{H}](\mathrm{HIRES})>-3.3 \mathrm{dex}$, the third is the only one that does not yet have a high resolution spectrum. It is, however, an C-star with extremely strong $\mathrm{CH}$, and therefore is very likely to have $[\mathrm{Fe} / \mathrm{H}]>-3.5 \mathrm{dex}$ based on our earlier work described in [16]. We assume for present purposes that this is the case; a HIRES spectrum to be obtained shortly should confirm this.

Two of the stars listed in the table are rediscoveries of previously known EMP stars found by the HK Survey [6]. In the end, after all this effort, we have found only two new stars with $[\mathrm{Fe} / \mathrm{H}](\mathrm{HIRES})<-3.5 \mathrm{dex}$, out of $\sim 1550$ follow up spectra.

Genuine EMP stars are very rare, and the EMP tail of the HES metallicity distribution function is heavily contaminated with stars which because of observational or analysis errors have landed up there, as well as with C-stars whose metallicity has been underestimated [see $16]$ and with some spurious objects.

Algorithms currently in use with SEGUE do not attempt to assign a metallicity based on $\mathrm{H} \delta$ and the Ca II $\mathrm{K}$ line to very $\mathrm{C}$-rich stars. This is a big step forward. However, the extreme low metallicity end of any MDF generated from HES follow up spectra is likely to be heavily contaminated with additional higher metallicity objects which through various issues, including observational errors, end up getting assigned a spurious very low 


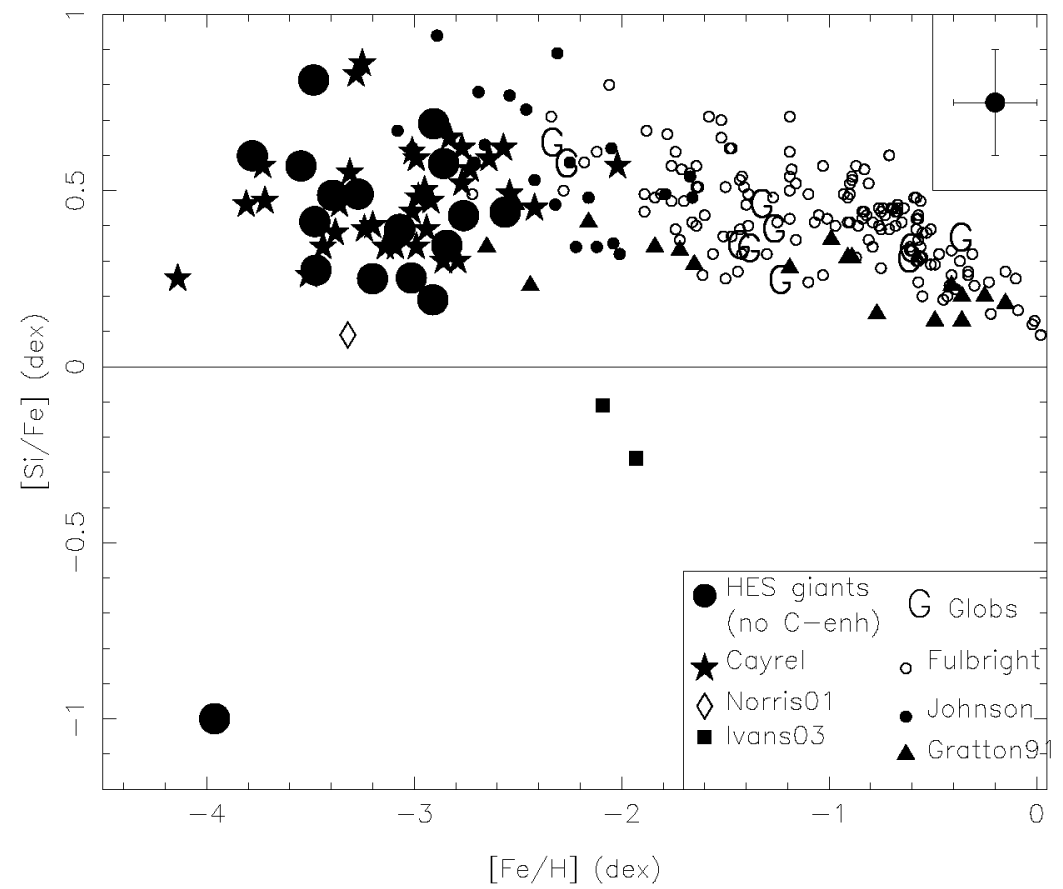

FIGURE 1. [Si/Fe] is shown for all of the candidate EMP stars with HIRES spectra analyzed by the 0Z project to date, including the present sample. C-rich stars are not shown. The solid horizontal line denotes the Solar ratio. The plot includes well studied Galactic globular clusters, mostly from analyses by J. Cohen and her collaborators, as well as samples of halo field stars from the sources indicated on the symbol key in the lower right of the figure. Note the highly anomalous position of HE1424-0241, the only star with $[\mathrm{Si} / \mathrm{Fe}]<<0$ dex.

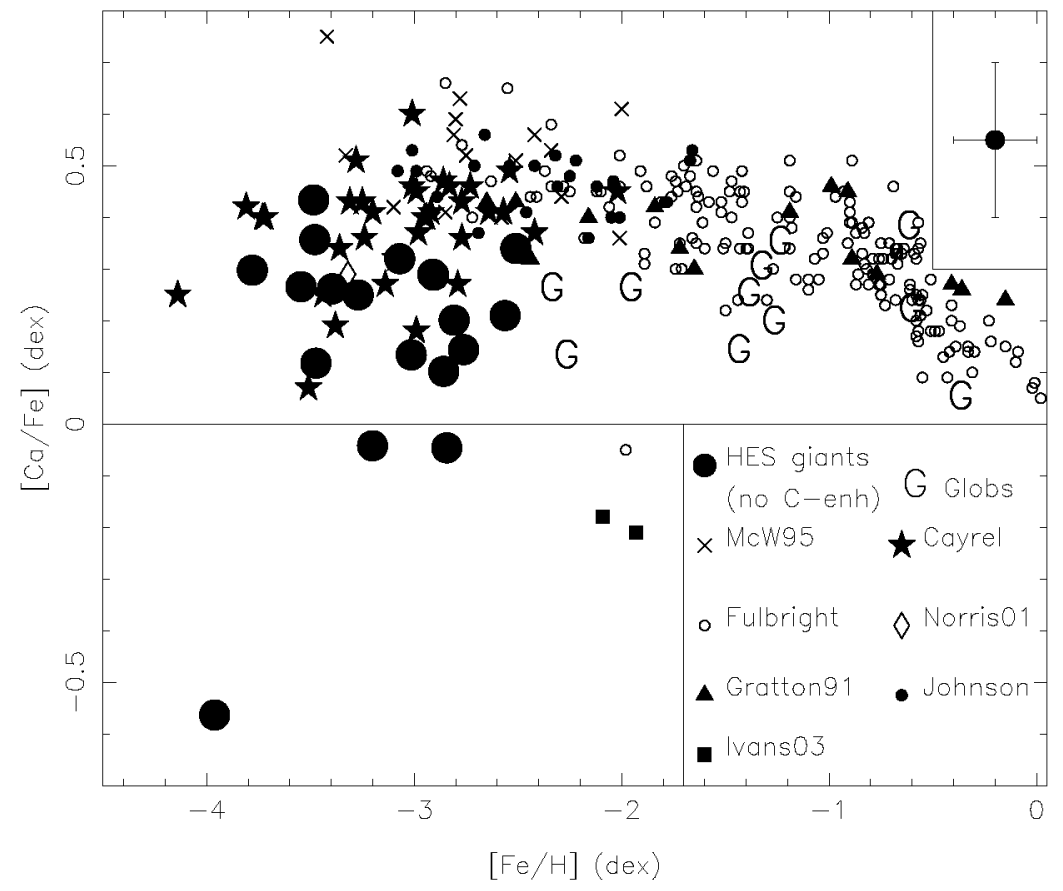

FIGURE 2. The same as Fig. 1 for $[\mathrm{Ca} / \mathrm{Fe}]$. 
TABLE 1. Status of Stars From Raw 0Z Survey With $[\mathrm{Fe} / \mathrm{H}](\mathrm{HES})<-3.5 \mathrm{dex}$

\begin{tabular}{lrrl}
\hline Star Name & {$[\mathbf{F e} / \mathbf{H}](\mathbf{H E S})$} & {$[\mathbf{F e} / \mathbf{H}](\mathbf{H I R E S})$} & \\
\hline HE1 159-0525 & -3.52 & $? ?$ & Extreme C-star, [Fe/H](HES) prob too low \\
HE1150-0428 & -3.57 & -3.27 & C-star \\
HE2209 & -3.57 & -3.47 & MIKE spectrum \\
HE1031-0020 & -3.61 & -2.88 & C-star \\
HE0122 & -3.63 & -2.80 & \\
HE0305-5442 & -3.68 & -3.56 & CS22968-014 rediscovery, $[\mathrm{Fe} / \mathrm{H}]$ from Cayrel et al (2004) \\
HE0313 & -3.72 & -3.63 & MIKE spectrum \\
HE0911-0512 & -3.84 & $\ldots$ & M dwarf \\
HE2323-0256 & -3.96 & -3.78 & CS22949-037 rediscovery \\
HE1432 & -4.43 & -2.51 & \\
HE0208-5335 & -4.14 & $\ldots$ & QSO (Ian Thompson) \\
HE1030+0137 & -4.37 & $\ldots$ & M dwarf \\
\hline
\end{tabular}

\section{$[\mathrm{Fe} / \mathrm{H}](\mathrm{HES})$.}

We now have HIRES observations in hand for essentially all of the 16 stars from our $0 \mathrm{Z}$ project with $-3.5<[\mathrm{Fe} / \mathrm{H}](\mathrm{HES})<-3.3 \mathrm{dex}$. Of the six analyzed thus far, only three have $[\mathrm{Fe} / \mathrm{H}](\mathrm{HIRES})<-2.8 \mathrm{dex}$. We are in the process of analyzing the rest of these to see what fraction of those in the regime of $[\mathrm{Fe} / \mathrm{H}](\mathrm{HES})$ between -3.5 and -3.3 dex are genuine. In the meantime, we urge caution in looking at the low-metallicity tail of any preliminary MDF from the HES in which the metallicities of stars at $[\mathrm{Fe} / \mathrm{H}]<-3.0$ dex are based on moderate-resolution spectra rather than high-resolution spectroscopy.

\section{ACKNOWLEDGMENTS}

This work is based in part on observations obtained at the W.M. Keck Observatory, which is operated jointly by the California Institute of Technology, the University of California, and the National Aeronautics and Space Administration. Some of the data presented herein were obtained at the Palomar Observatory. We are grateful to the many people who have worked to make the Keck Telescope and HIRES a reality and to operate and maintain the Keck Observatory. J.G.C. is grateful to NSF grant AST-0507219 for partial support. N.C. is a Research Fellow of the Royal Swedish Academy of Sciences supported by a grant from the Knut and Alice Wallenberg Foundation. He also acknowledges financial support from Deutsche Forschungsgemeinschaft through grants Ch 214/3 and Re 353/44. We thank Tim Beers for providing us with $[\mathrm{Fe} / \mathrm{H}](\mathrm{HES})$ values using the most recent version of his codes.

\section{REFERENCES}

1. T. C. Beers and N. Christlieb, Ann.Revs.Astron.\& Astrophys 43531 (2005)

2. Wisotzki, L., Christlieb, N., Bade, N.,Beckmann, V., Köhler, T., Vanelle, C. \& Reimers, D., $A \& A 35877$ (2000)

3. N. Christlieb, Rev. Mod. Astron. 16191 (2003)

4. Cohen, J. G. et al., $A p J$ in press (2008)

5. T. C. Beers, S. Rossi, J. E. Norris, S. Ryan \& T. Shefler, AJ 117981 (1999)

6. T. C. Beers, G. W. Preston. \& S. Shectman, AJ 1031987 (1992)

7. S. Rossi, T. C. Beers, C. Sneden, T. Sevastyanenko, J. Rhee, \& B. Marsteller $A J 1302804$ (2005)

8. Y. S. Lee et al., BAAS 298168.15 (2006)

9. Cohen, J. G., Christlieb, N., McWilliam, A., Shectman, Thompson, I., Wasserburg, G. J., Ivans, I., Dehn, Karlsson, T. \& Melendez, J., 2004, ApJ 6121107 (2004)

10. R. Cayrel, R. et al., $A \& A 4161117$ (2004)

11. E. Arnone, S. G. Ryan, D. Argast, J. E. Norris \& T. C. Beers, $A \& A 430507$ (2005)

12. Spite, M. et al., $A \& A 430655$ (2005)

13. J. G. Cohen, N. Christlieb, Y. Z. Qian \& G. J. Wasserburg, ApJ $\mathbf{5 8 8} 1082$ (2003)

14. J. G. Cohen, A. McWilliam, N. Christlieb, S. Shectman, I. Thompson, J. Melendez, L. Wisotzki, \& E. Reimers, ApJL 659 L161 (2007)

15. Fulbright, J. P., McWilliam, A. \& Rich, R. M., ApJ 661 $1152(2007)$

16. Cohen, J. G. et al., ApJL 633 L109 (2005) 